\title{
Antineutrophil cytoplasmic antibody and vasculitis: much more than a disease marker
}

\author{
Vivette D’Agati \\ Department of Pathology, College of Physicians and Surgeons, Columbia University, New York, New York, USA \\ J. Clin. Invest. 110:919-921 (2002). doi:10.1172/JCI200216699.
}

Many forms of necrotizing small vessel vasculitis manifest glomerulonephritis, pulmonary hemorrhage and cutaneous purpura. Because the histologic appearance of the vasculitic lesions is similar in these conditions, early pathologists and clinicians resorted to categorizing small vessel vasculitides based on vessel size and systemic distribution. Not surprisingly, due to the tremendous overlap in clinical-pathologic features, these early classifications were woefully inadequate and perpetuated enduring confusion between the different nosologies adopted by internists, rheumatologists, nephrologists, and dermatologists.

The introduction of immunofluorescence microscopy in the 1960 s provided a major advance by uncovering three major immunopathologic categories of vasculitis. The demonstration of linear staining of alveolar and glomerular basement membranes (GBMs) in Goodpasture Syndrome led to the identification of circulating anti-GBM antibodies that cross-react with alveolar basement membrane, producing a pulmonary-renal syn-

\footnotetext{
Address correspondence to: Vivette D'Agati, Department of Pathology, College of Physicians and Surgeons, Columbia

University, 630 West 168th Street, New York, New York 10032, USA.

Phone: (212) 305-7460; Fax: (212) 342-5380;

E-mail: vdd1@columbia.edu.

Conflict of interest: No conflict of interest has been declared.

Nonstandard abbreviations used: glomerular basement membrane (GBM); antineutrophil cytoplasmic autoantibody (ANCA); perinuclear ANCA (P-ANCA); cytoplasmic ANCA (C-ANCA); myeloperoxidase (MPO); proteinase 3 (PR3); mitogen-activated protein kinase (MAPK); extracellular signal-regulated kinase (ERK); intercellular adhesion molecule-1 (ICAM-1); vascular cell adhesion molecule-1 (VCAM-1); Graves disease (GD).
}

drome. In the second major category of small vessel vasculitis, granular deposits of immunoglobulin and complement could be demonstrated in vessel walls and glomeruli. IgAdominant deposits were identified in the cutaneous vessels and glomeruli of patients with Henoch Schonlein purpura, deposits of IgG-IgM in patients with mixed cryoglobulinemia, and IgG-dominant deposits in patients with lupus vasculitis and septic vasculitis. However, there was a third category of vasculitis (including microscopic polyangiitis, Wegener granulomatosis, Churg-Strauss syndrome, and renal-limited pauci-immune crescentic glomerulonephritis) for which few if any immune deposits could be identified in target tissues. Unraveling the pathogenesis of these pauci-immune vasculitides posed a special challenge, because this group defied existing paradigms of in situ or passive immune complex deposition. In the absence of demonstrable immune deposits, it was naturally assumed that the vascular injury was of a cellular nature, but it would be many years before attention focused on the neutrophil.

\section{Antineutrophil cytoplasmic antibodies come of age}

A breakthrough in our understanding of the pathogenesis of small vessel vasculitis and pauci-immune glomerulonephritis came in 1982, when Davies and colleagues reported the identification of an antibody that reacted with human neutrophils in a small cohort of patients with necrotizing glomerulonephritis (1). These investigators stumbled upon their discovery by chance while testing for anti-nuclear antibodies using an indirect immunofluorescence assay that employed human neutrophils as substrate. This curious observation received little attention until 1985, when a group of investigators found a strong association between antineutrophil cytoplasmic antibodies and active Wegener granulomatosis (2).

The next task was to elucidate the nature of the antigenic targets recognized by antineutrophil cytoplasmic antibodies (ANCAs). Two major patterns of ANCA immunostaining, perinuclear and cytoplasmic, were observed by indirect immunofluorescence after application of patient serum to a substrate of alcohol-fixed human neutrophils. The perinuclear (P) pattern corresponds to ANCA with specificity for myeloperoxidase (P-ANCA), whereas the cytoplasmic (C) pattern corresponds to ANCA with specificity for proteinase-3 (C-ANCA). Both antigenic targets are now recognized to be cytoplasmic and to reside in the azurophilic granules and lysosomes of neutrophils and monocytes, but because myeloperoxidase redistributes to the nuclear membrane during sample preparation, it gives a perinuclear pattern in alcohol-fixed, but not formalin-fixed, neutrophils.

In the early 1990s, investigators focused their efforts on correlating the pattern and antigenic specificity of ANCA with distinct clinical-pathologic syndromes. It soon became clear that C-ANCA is more commonly detected in patients with Wegener granulomatosis, whereas P-ANCA predominates in patients with microscopic polyangiitis, renal-limited pauci-immune crescentic glomerulonephritis, and Churg-Strauss syndrome. This novel serologic marker for pauci-immune vasculitis could be detected in up to $90 \%$ of patients with active vasculitis. It required only a small leap to hypothesize that an autoanti- 
body with such sensitivity for pauciimmune vasculitis might be a major pathogenetic player.

\section{From serologic marker to in vitro studies}

ANCA presented a highly attractive candidate mediator of necrotizing pauci-immune vasculitis because it could account for a destructive inflammatory process that proceeds through mechanisms centered on neutrophil activation in the absence of tissue deposits of immune reactants. How ANCAs might reach target antigens sequestered in the cytoplasm of neutrophils and monocytes was uncertain, but in vitro evidence that ANCAs bind to trace amounts of myeloperoxidase (MPO) and proteinase-3 (PR3) expressed on the surface of TNF-primed neutrophils and monocytes suggested a possible route (3). Cytokine priming stimulates redistribution of such granule contents to the neutrophil surface, where they are free to interact with ANCAs by both $\mathrm{Fab}^{\prime} 2$ and $\mathrm{Fc}$ engagement (4). During periods of active vasculitis, elevated circulating levels of TNF can be measured in patient serum, and both protein and mRNA levels of TNF are increased at the sites of vasculitis (5). Patients with active vasculitis have enhanced TNF expression in peripheral blood mononuclear cells and elevated serum levels of the soluble receptors, TNF-R55 and TNF-R7 (6). Neutrophil activation markers, such as CD66b, CD64, and CD63, are upregulated on primed circulating neutrophils in human vasculitis and correlate with disease activity (7). The in vivo correlate of the priming event in vitro is likely to be an intercurrent viral or bacterial infection, usually in the upper respiratory tract. Such infections commonly precede the onset or recrudescence of ANCA-associated vasculitis and may explain the seasonal variations in incidence.

Primed neutrophils that interact with ANCA are stimulated to become fully activated and undergo a respiratory burst with release of toxic oxygen radicals and lytic enzymes on endothelial surfaces (3). The signals involved in neutrophil respiratory burst have been recently dissected and include p38 mitogen-activated protein kinase (MAPK) and extracellular signal-regulated kinase (ERK), as well as phos- phatidylinositol 3 kinase control systems (8). Endothelial killing in vitro by primed neutrophils activated by ANCA involves adhesion of the neutrophils to endothelium via $\beta 2$ integrins and upregulation of intercellular adhesion molecule-1 (ICAM-1) and vascular cell adhesion molecule-1 (VCAM-1). Recent evidence suggests that TNF-primed neutrophils undergo accelerated and dysregulated apoptosis and that ANCA-antigen is expressed on the surface of these apoptotic neutrophils at tissue sites where leukocytoclasia can augment inflammatory injury (9). ANCA also may react with antigens that are released into the circulation or tissue microenvironment to form soluble immune complexes that may be adsorbed onto vessel walls, thereby amplifying the inflammatory process. PR3 may also be expressed, albeit at low levels, on the surface of endothelial cells where interaction with ANCA could cause in situ immune complex formation (10).

\section{From in vitro to in vivo systems}

The large body of in vitro data supporting a potential pathogenic role of ANCA has been crying out for an in vivo system to prove causality. Some skeptics have argued that ANCA is merely an epiphenomenon of neutrophil activation, rather than a primary pathogenic mediator. Establishment of an animal model has proven extremely difficult. Although several in vivo systems support ANCA's pathogenicity, most such models involve glomerulonephritis and implicate ANCA as a cofactor, not an independent cause of the disease. For example, ANCAs have been identified in rats treated with mercury chloride, in MRL$l p r / l p r$ mice, and in SCG $/ \mathrm{Kj}$ mice, where the role of ANCA is difficult to tease out from the complex background of polyclonal B cell activation (11). Likewise, Kobayashi et al. induced rat nephrotoxic serum nephritis with subnephritic doses of anti-GBM antibody by addition of rabbit anti-rat MPO (12), and Heeringa et al. showed that immunization of rats with human MPO could provoke an acute glomerulonephritis under conditions that would otherwise lead only to mild antiGBM disease (13). In studies by Brouwer et al., immunization of rats with human MPO induced antibodies to both human and rat MPO (14). When the kidneys of these rats were perfused with human neutrophil extracts, they developed a crescentic glomerulonephritis (14). However, the presence of glomerular immune deposits of $\mathrm{IgG}$, $\mathrm{MPO}$ and C3 in this model suggests more complex mechanisms that are at variance with the pauci-immune nature of the human disease.

The present article by Xiao et al., from the laboratories of J. Charles Jennette and Ronald J. Falk, provides the first convincing evidence that ANCAs are sufficient to cause systemic pauciimmune vasculitis and glomerulonephritis in vivo (15). These authors use two major strategies to demonstrate a direct causal link between ANCA and the development of glomerulonephritis. In the first, MPO knockout mice were immunized with murine MPO and developed circulating anti-MPO. Adoptive transfer of anti-MPO splenocytes into immunedeficient Rag2-/- mice (which lack functioning $\mathrm{B}$ - and T-lymphocytes) resulted in circulating anti-MPO ANCA and the development of a crescentic glomerulonephritis, extrarenal vasculitis and pulmonary capillaritis. By contrast, Rag2-/- mice receiving anti-BSA splenocytes or control splenocytes develop a milder form of immune complex glomerulonephritis with endocapillary hypercellularity, but no necrosis or crescents. In a second set of experiments, the authors showed that purified anti-MPO IgG, injected intravenously into Rag2 ${ }^{-/}$ mice or wild-type mice, induces a pauci-immune, focal, necrotizing and crescentic glomerulonephritis and systemic vasculitis that closely recapitulate the human disease. In the passive transfer experiments, the nature of the background immune complex disease that developed in Rag2-/- mice receiving either anti-MPO or anti-BSA splenocytes is unclear, but this relatively non-specific response may represent a form of graft vs. host disease. The ability of anti-MPO splenocytes to incite crescentic transformation of immune-mediated glomerulonephritis is congruent with the observation in humans that ANCA can promote a crescentic phenotype in a variety of immune complex-mediated glomerular diseases, such as lupus nephritis or IgA nephritis. The development of vasculitis and glomerulonephritis following intravenous administration of 
anti-MPO in immunodeficient mice indicates that anti-MPO can produce vasculitis without the participation of $\mathrm{T}$ or B cells. The stage is now set to prove that the glomerulonephritis is indeed neutrophil-mediated.

\section{Future directions}

Armed with the first convincing animal model of ANCA-vasculitis, we are now poised to test the predictions of in vitro experiments in a powerful in vivo system. It will be important to confirm that the glomerulonephritis is neutrophil-mediated and proceeds through mechanisms of neutrophil activation and degranulation. Neutrophil priming by cytokines such as TNF prior to anti-MPO administration would be expected to increase the severity of the glomerulonephritis, allowing lower doses of anti-MPO to initiate nephritis. Gene microarrays might be used to study the program of gene activation at particular disease sites. The role of specific effector cells and molecules can be tested by their selective depletion or blockade. Of particular interest will be the question of FcR engagement, which can be tested in FcR knockout mice, and the potential participation of macrophages, NK cells, and T cells in the inflammatory process. Indeed, the greater severity of the glomerulonephritis induced by anti-MPO splenocytes compared to anti-MPO IgG suggests a role for $\mathrm{T}$ cells in the augmentation of immune injury (16).

The potential role of $\mathrm{T}$ cells raises the question of whether ANCA-associated vasculitis should now be included on the growing list of recognized autoimmune diseases. In most such diseases, specific autoantibodies, such as those to the $\beta$ islet cells (in type 1 diabetes) or to the thyroid epithelium (in Hashimoto thyroiditis), eliminate their target cell by cellular cytotoxicity. Here, however, we may be dealing with a different type of autoimmune condition, one where tissue destruction results from neutrophil activation per se, and where the role of autoantibodies is specifically to engage these inflammatory cells. Among well studied autoimmune con- ditions, some parallels can be drawn to Graves disease (GD) (see ref. 17 for review). In pauci-immune vasculitis, as in GD, autoantibodies activate a target cell population (neutrophils and thyroid follicular epithelial cells, respectively) and stimulate specific cell functions. The autoantibodies in GD activate their target cell by ligating a specific cell surface receptor, the thyrotropin receptor (TSHR). In contrast, the only receptor-mediated interaction that has been documented in ANCAassociated vasculitis occurs through Fc receptor engagement on the surface of the neutrophil. Nevertheless, in both cases, the presence of autoantibody stimulates specific signaling events that leave the cell in a hyper-activated functional state.

We now know that the passive transfer of ANCA autoantibody is sufficient to induce disease, but it remains to be discovered how the production of autoantibodies to neutrophil antigens might be triggered. Standard definitions of autoimmune disease require activation of autoreactive $\mathrm{T}$ cells, and indeed, autoreactive $\mathrm{T}$ cell clones might drive autoantibody production and contribute to late inflammatory sequelae in the vasculature and tissues of affected individuals. The occurrence of ANCA-vasculitis as a drug reaction to propylthiouracil, pimagedine, and minocycline suggests that molecular mimicry may promote this reaction in some circumstances (18). The wide variation among normal individuals in the percentage of neutrophils that stably express surface PR3 suggests that genetically determined polymorphisms could affect susceptibility to this disease (19). Greater understanding of the potential participation of specific effector cells, identification of individuals at risk for autoantibody production, and elucidation of the priming events in man will allow future design of more effective preventive and therapeutic strategies.

1. Davies, D.J., Moran, J.E., Niall, J.F., and Ryan, G.B. 1982. Segmental necrotizing glomerulonephritis with antineutrophil antibody: possible arbovirus aetiology? Br. Med.J. 285:606.

2. van der Woude, F.J., et al. 1985. Autoantibodies against neutrophils and monocytes: tool for diagnosis and marker of disease activity in Wegener's granulomatosis. Lancet. 1:425-429.

3. Falk, R.J., Terrell, R.S., Charles, L.A., and Jennette, J.C. 1990. Anti-neutrophil cytoplasmic autoantibodies induce neutrophils to degranulate and produce oxygen radicals in vitro. Proc. Natl. Acad. Sci. USA. 87:4115-4119.

4. Mulder, A.H., Heeringa, P., Brouwer, E., Limburg, P.C., and Kallenberg, C.G. 1994. Activation of granulocytes by anti-neutrophil cytoplasmic antibodies (ANCA): a Fc gamma RII-dependent process. Clin. Exp. Immunol. 98:270-278.

5. Noronha, I.L., Kruger, C., Andrassy, K., Ritz, E. and Waldherr, R. 1993. In situ production of TNF- $\alpha$, IL- $1 \beta$ and IL-2R in ANCA-positive glomerulonephritis. Kidney Int. 43:682-692.

6. Nassonov, E., et al. 1997. Serum concentrations of neopterin, soluble interleukin 2 receptor and soluble tumor necrosis factor receptor in Wegener's granulomatosis. Br. J. Rheumatol. 24:666-670.

7. Muller Kobold, A.C., Mesander, G., Stegeman, C.A., Kallenberg, C.G.M., and Tervaert, J. 1998. Are circulating neutrophils intravascularly activated in patients with anti-neutrophil cytoplasmic antibody (ANCA)-associated vasculitides? Clin. Exp. Immunol. 114:491-499.

8. Kettritz, R., et al. 2002. Phosphatidylinositol 3-kinase controls antineutrophil cytoplasmic antibodies-induced respiratory burst in human neutrophils. J. Am. Soc. Nephrol. 13:1740-1749.

9. Harper, L., Ren, Y., Savill, J., Adu, D., and Savage, C.O.S. 2000. Antineutrophil cytoplasmic antibodies induce reactive oxygen-dependent dysregulation of primed neutrophil apoptosis and clearance by macrophages. Am. J. Pathol. 157:211-220.

10. Mayet, W.J., Schwarting, A., and Meyer zum Buschenfelde, K.H. 1994. Cytotoxic effects of antibodies to proteinase 3 (C-ANCA) on human endothelial cells. Clin. Exp. Immunol. 97:458-465.

11. Kinjoh, K., Kyogoku, M., and Good, R.A. 1993. Genetic selection for crescent formation yields mouse strain with rapidly progressive glomerulonephritis and small vessel vasculitis. Proc. Natl. Acad. Sci. USA. 90:3413-3417.

12. Kobayashi, K., Shibata, T., and Sugisaki, T. 1995 Aggravation of rat nephrotoxic serum nephritis by anti-myeloperoxidase antibodies. Kidney. Int 47:454-463.

13. Heeringa, P., et al. 1996. Autoantibodies to myeloperoxidase aggravate mild anti-glomerular basement membrane-mediated glomerular injury in the rat. Am. J. Pathol. 149:1695-1706.

14. Brouwer, E., et al. 1993. Antimyeloperoxidaseassociated proliferative glomerulonephritis: an animal model. J. Exp. Med. 177:905-914.

15. Xiao, H., et al. 2002. Antineutrophil cytoplasmic autoantibodies specific for myeloperoxidase cause glomerulonephritis and vasculitis in mice. J. Clin. Invest. 110:955-963. doi:10.1172/JCI200215918.

16. Clayton, A.R., and Savage, C.O.S. 2000. Evidence for the role of $T$ cells in the pathogenesis of systemic vasculitis. Arthritis Res. 2:260-262.

17. Rapoport, B., and McLachlan, S.M. 2001. Thyroid autoimmunity. J. Clin. Invest. 108:1253-1259. doi:10.1172/JCI200114321.

18. ten Holder, S.M., Joy, M.S., and Falk, R.J. 2002. Cutaneous and systemic manifestations of drug-induced vasculitis. Ann. Pharmacother. 36:130-147.

19. Witko-Sarsat, V., et al. 1999. A large subset of neutrophils expressing membrane proteinase 3 is a risk factor for vasculitis and rheumatoid arthritis. J. Am. Soc. Nephrol. 10:1224-1233. 